\title{
Registration of the number of macules in paucibacillary leprosy for evaluation of early diagnosis and individual prognosis
}

\author{
M DEGUERRY*, E DECLERCQ*, C MISSON*, \\ C VELLUT $\dagger \&$ F BERTRAND* \\ *Department of Epidemiology, School of Public Health, Catholic \\ University of Louvain, 1200 Brussels, Belgium; †Hemerijckx \\ Government Leprosy Centre, Polambakkam 603309, Tamil Nadu, \\ India
}

Accepted for publication 24 April 1989

\begin{abstract}
Summary The number of macules is usually registered at diagnosis in the first clinical examination of leprosy patients. The question studied here is whether this practice is of any interest as an indicator of the precocity of detection or the prognosis. The study is based on the 26,996 paucibacillary patients detected from 1957 to 1982 in Polambakkam Leprosy Centre (South India) for whom the number of macules and disability status are assessed and registered.

Several observations suggest that the proportion of single-macule patients among the newly detected cases is a more sensitive indicator than the proportion of new patients with disabilities for the evaluation of the delay between onset of the disease and detection. Its use could be especially helpful for programmes running for several years, when it becomes difficult to observe significant variations in the proportion of patients with disabilities.

Regarding the prognosis value of the number of macules, inactivation and relapse probabilities were calculated. Regularity of treatment is found to be a better predictor of early inactivation than the number of macules, while relapse probabilities are more affected by the number of macules.
\end{abstract}

\section{Introduction}

Evaluation should be an effective part of every leprosy control programme, and should be based on the collection and the analysis of appropriate and reliable data.

The OMSLEP information system is now used more and more for evaluation in the field. ${ }^{1}$ It has been created at the request of the WHO in an attempt to standardize and make comparable the data collected, and to limit them to those really useful for decision-making. However, many health managers want to adapt it to the local situation and to collect some additional data. One of the variables often added to the individual patient form is the number of macules at detection for paucibacillary cases. Information concerning the number and evolution of macules should be collected in the patient clinical file at least annually to be useful for individual follow-up. The usefulness of this data as an epidemiological indicator, has never been properly evaluated. If limited 
to the number of macules at detection, this information could only be worth reporting if it can help to build either: a more sensitive indicator of early detection than the proportion of patients with disabilities among the newly detected cases; or an indicator of prognosis, either for the duration of treatment until inactivation of the lesions, or for the risk of relapse.

Thus this study has two objectives. First, to study whether the proportion of single-macule patients among newly detected cases is a more sensitive indicator of early diagnosis than the presence of disability. This objective is only verified if the several-macules stage is preceded by the single-macule stage and if the delay to go from one to several macules is shorter than the delay necessary to develop disabilities. The second objective is to evaluate the prognosis value of the number of macules at detection. It will be reached by comparing inactivation and relapse probabilities between single- and several-macule patients.

\section{Material and methods}

This study is based on the data routinely collected in the Polambakkam Leprosy Control Programme, in South India, where 47,068 patients were detected between 1955 and 1982. Among these patients eligible for the present study were those:

of the paucibacillary type of the disease (either clinical tuberculoid type or borderline type with negative bacteriological status);

detected from 1957 to 1982 , as the first 2 years showed too many missing values regarding the number of macules at detection;

never treated before; and

with a number of macules and presence of disabilities both assessed at detection.

According to these criteria 26,996 patients were selected for the analysis. For the second objective, i.e. evaluation of the prognosis value of the number of macules, attendance to treatment had also to be known from detection to either inactivation or cure. This was the case for 26,106 patients.

Information on the number of macules and the presence of disabilities was only registered at detection, and not at the subsequent follow-ups. The term 'macule' was actually used for all kinds of patches, either flat or partly or wholly elevated, that are characteristic of leprosy. The number of macules at detection was recorded in only two categories: one macule, and more than one.

Disabilities included bone lesions, claw hands, drop feet, facial lesions and ulcers. Anaesthesia is not taken into account. Distinction was made between index and contacts patients. Patients living in the same household as an already registered case were considered as contacts. This distinction was necessary because:

mean age may be different for the contact and the noncontact populations; the contact population is more likely to be infected; and the contact population is more frequently and regularly examined, leading to a higher probability of early detection.

Contact status was unknown for 554 patients. Standard treatment was dapsone monotherapy, to be continued after inactivation for a consolidation period whose duration was based on the WHO recommendations. ${ }^{6}$ Attendance for treatment is estimated as the number of attended sessions of treatment divided by the number of organized sessions and expressed in two groups ( $\geq 75 \%$ and $<75 \%$ ). Clinical status of the patients was recorded annually.

In the study area, inactivation was defined as 'complete subsidence of thickening and erythema in the patches resulting in the wrinkling and scanning of lesion with partial or complete return to sensation and complete subsidence of activity in the peripheral and trunk nerves'. Relapses concern patients who developed new signs and symptoms of the disease after stopping therapy.

To evaluate the validity of the number of macules at detection as an indicator of early diagnosis 
and prognosis, a longitudinal study would be the appropriate methodology. Unfortunately, information concerning the number of macules and the disabilities was registered at detection only. Consequently, for the first objective (early diagnosis) we had to make do with a cross-sectional analysis to get hints on the evolution of the number of macules. For the second objective (prognosis), data concerning clinical status was collected at yearly follow-ups, enabling us to perf orm the appropriate longitudinal study. This was based on the actuarial or life-table method. ${ }^{2}$

\section{Results}

\section{EARLY DIAGNOSIS}

Distribution and mean age of the patients by number of macules and presence of disabilities at detection are shown in Table 1. The percentage of patients with disabilities is 3.7 times higher in the several-macule than in the single-macule patients. The difference of mean age observed between several and single-macule patients $(1 \cdot 8-3 \cdot 9$ years $)$ is much smaller than the one observed between patients with and without disabilities (10.5-12.6 years). Mean age for each disability status, is lower for the single than for the several-macule patients. This observation is verified throughout the whole period for both index and contact groups, as shown in Figure 1. Due to small numbers, the distinction between index and contact was not possible for the patients with disabilities. Tables 2

Table 1. Distribution and mean age of the patients by the number of macules and presence of disabilities at detection

\begin{tabular}{lccccc}
\hline & \multicolumn{2}{c}{ Single-macule patients } & & \multicolumn{2}{c}{ Several-macule patients } \\
\cline { 2 - 3 } \cline { 5 - 6 } \cline { 5 - 5 } & Without disability & With disabilities & & Without disability & With disabilities \\
\hline $\begin{array}{l}\text { No. } \\
\%\end{array}$ & 16,051 & 259 & & 10,050 & 636 \\
$\begin{array}{l}\text { Mean } \\
\text { age }\end{array}$ & $98 \cdot 4$ & 1.6 & & $94 \cdot 0$ & 6.0 \\
\hline
\end{tabular}

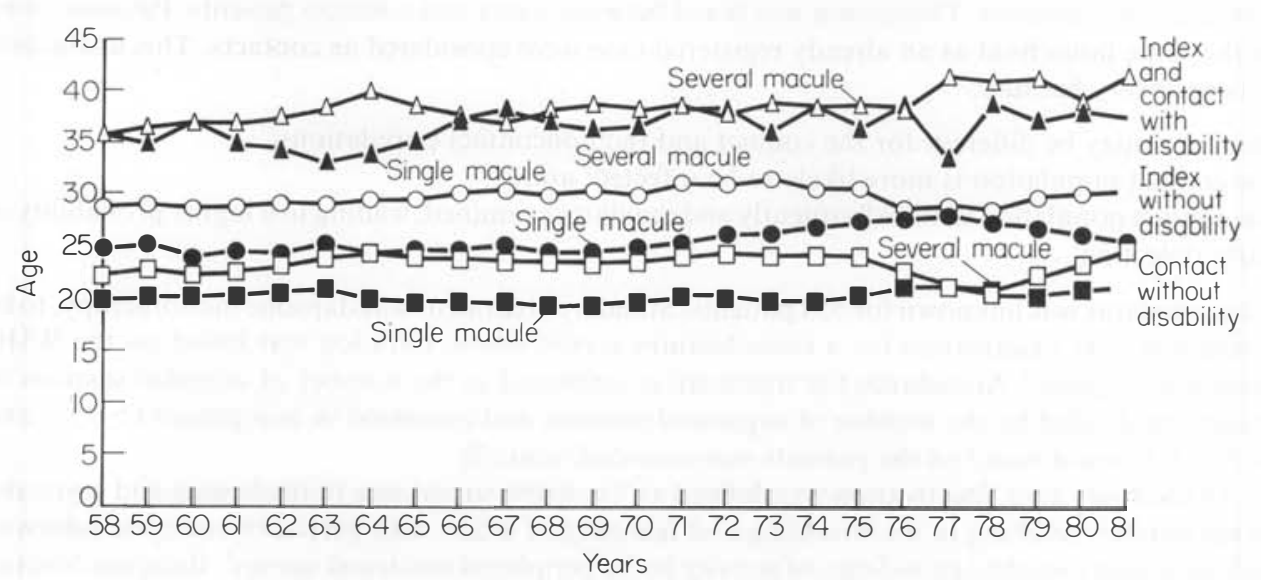

Figure 1. Three years moving average of the a ge of the patients according to the number of macules, the presence of disability and the contact status. 
and 3 show the distribution of the patients by number of macules, presence of disabilities and contact status. The proportion of several-macule patients is similar for both index and contact groups, while the proportion of patients with disabilities in the index group is two times higher than in the contact group. Figure 2 describes the trends of proportions of single-macule and disabled patients among newly detected cases, from 1957 to 1982 . The irregularities observed in 1960 in both curves reflect the extension of the leprosy control area, with a sudden detection of many old cases. For both curves, a significant slope could be demonstrated, using a logistic regression from 1961 onwards $(p<0.001$ for the disabilities and $p<0.001$ for the macules).

Table 2. Distribution of the patients by number of macules at detection and contact status*

\begin{tabular}{|c|c|c|c|c|c|c|}
\hline & \multicolumn{2}{|c|}{ Single-macule } & \multicolumn{2}{|c|}{ Several-macule } & \multicolumn{2}{|c|}{ Total } \\
\hline & Index & Contacts & Index & Contacts & Index & Contacts \\
\hline No. & 9,602 & 6,392 & 6,639 & 3,809 & 16,241 & 10,201 \\
\hline$\%$ & $59 \cdot 1$ & $62 \cdot 7$ & $40 \cdot 9$ & $37 \cdot 3$ & 100 & 100 \\
\hline
\end{tabular}

* Contact status was unknown for 554 patients.

Table 3. Distribution of the patients by presence of disabilities at detection and contact status*

\begin{tabular}{|c|c|c|c|c|c|c|}
\hline & \multicolumn{2}{|c|}{ Without disability } & \multicolumn{2}{|c|}{ With disabilities } & \multicolumn{2}{|c|}{ Total } \\
\hline & Index & Contacts & Index & Contacts & Index & Contacts \\
\hline $\begin{array}{l}\text { No. } \\
\%\end{array}$ & $\begin{array}{r}15,572 \\
95 \cdot 9\end{array}$ & $\begin{array}{l}9,997 \\
98 \cdot 0\end{array}$ & $\begin{array}{l}669 \\
4 \cdot 1\end{array}$ & $\begin{array}{r}204 \\
2 \cdot 0\end{array}$ & $\begin{array}{r}16,241 \\
100\end{array}$ & $\begin{array}{r}10,201 \\
100\end{array}$ \\
\hline
\end{tabular}

* Contact status was unknown for 554 patients.

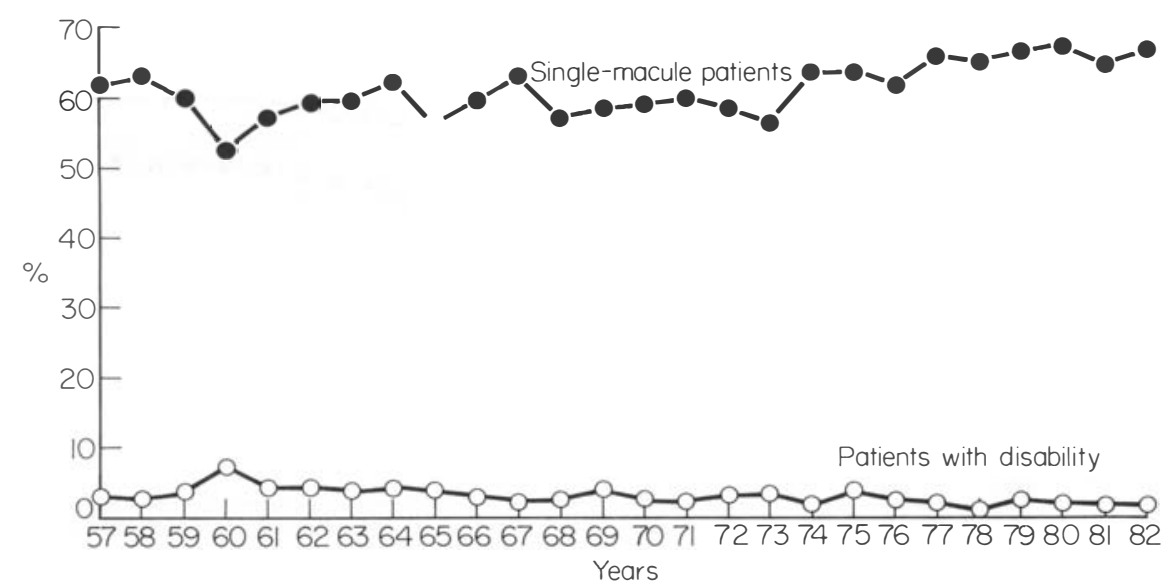

Figure 2. Annual evolution of the percentages of single-macule patients and of patients with disability. 


\section{PROGNOSIS}

Whatever their number of macules, patients with good attendance to treatment inactivate sooner than patients with poor attendance (Figure 3). Within each group of attendance, single-macule patients inactivate sooner than several-macule patients. Figure 4 shows relapse probabilities after inactivation by number of macules at detection. These probabilities are lower for single-macule patients, even after a long observation period. This is still true when results are displayed by attendance to treatment (Figure 5).

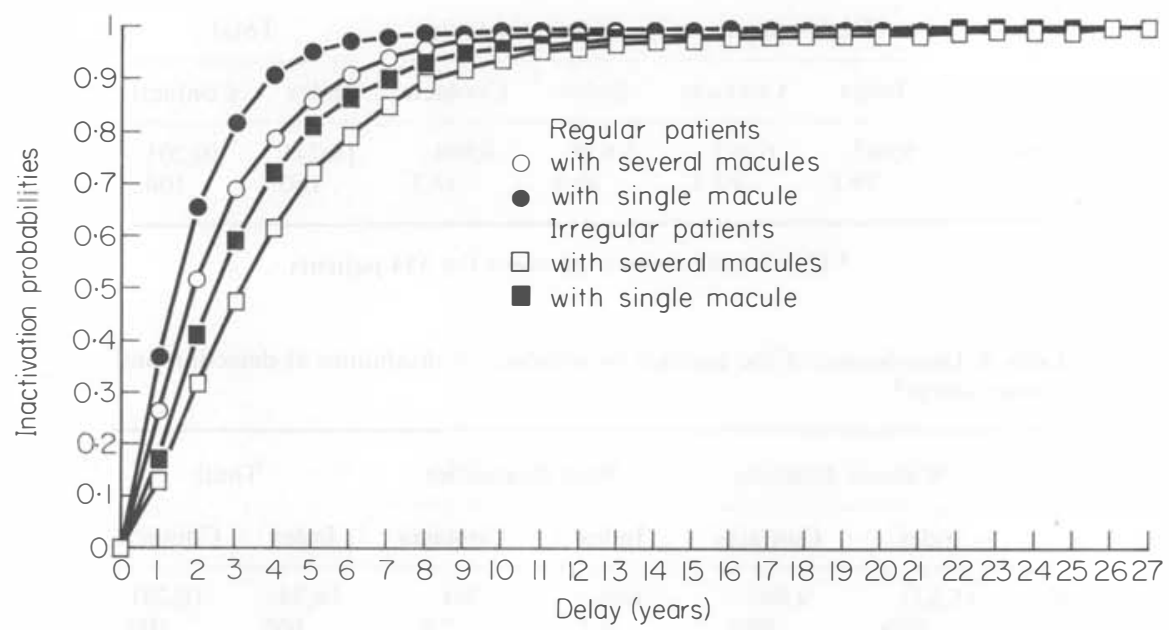

Figure 3. Cumulative inactivation probabilities from beginning of treatment, by number of macules at detection and attendance.

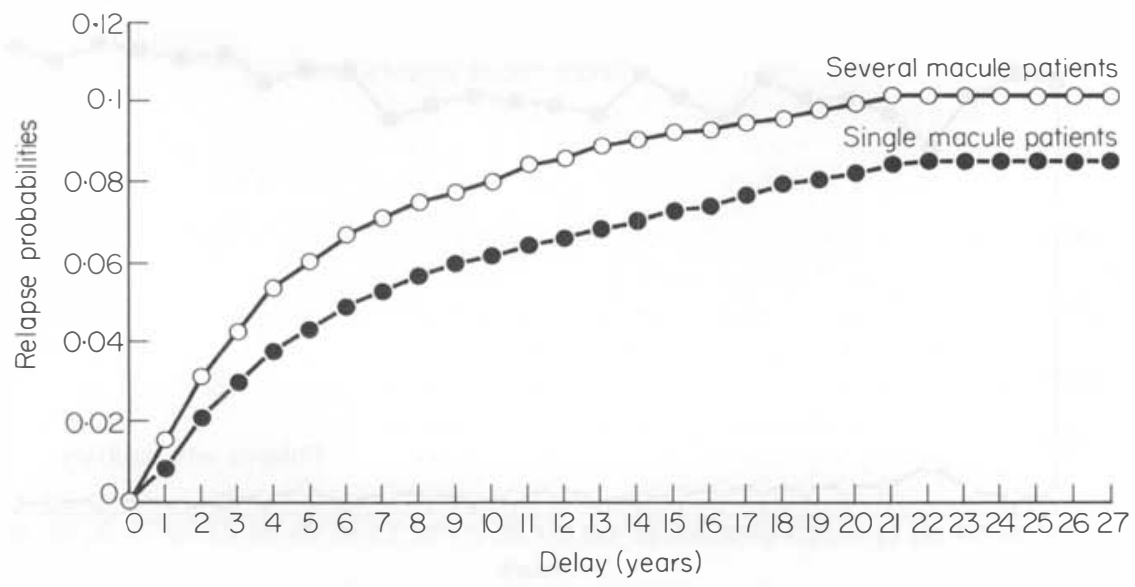

Figure 4. Cumulative relapse probabilities after inactivation, by number of macules at detection. 


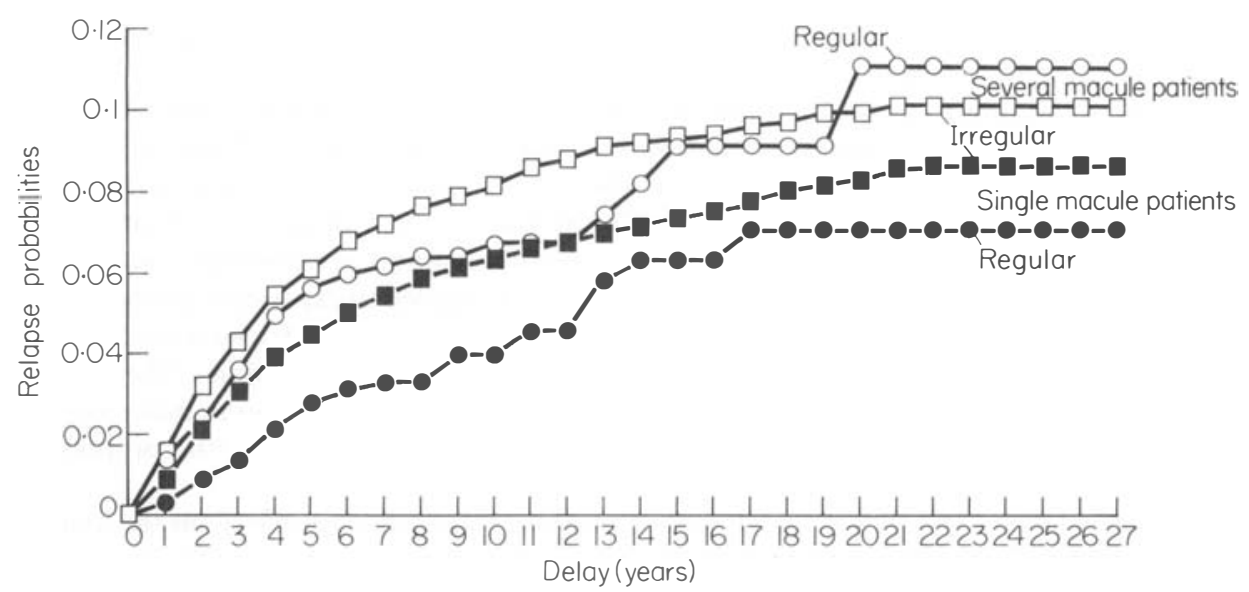

Figure 5. Cumulative relapse probabilities after inactivation, by number of macules at detection and attendance.

\section{Discussion}

\section{EARLY DIAGNOSIS}

The proportion of disabled among newly detected cases is widely accepted as an indicator of early diagnosis, ${ }^{5}$ early detected patients having a lower probability of presenting disabilities. Repeated observations $\mathrm{s}^{3.4}$ showed that the proportion of disabled patients decreases with the improvement of detection activities. For the proportion of single-macule patients among newly detected cases to be also accepted as an indicator of early diagnosis, the several-macule stage should be preceded by a single-macule stage. With the limitations of a cross-sectional study, one would then expect to make the following observations:

a higher proportion of single-macule patients among the patients without disability than among those with disabilities;

an increasing proportion of single-macule patients when the proportion of disabled among newly detected cases decreases over time;

a lower mean age at detection for the single-macule than for the several-macule patients; and a higher proportion of single-macule patients among the contacts than among the index, since the contact population is more frequently examined, and consequently detected earlier;

The actual observations reported in Tables 1 to 3 and Figures 1 and 2, are consistent with the expectations. One could object that the same observation could be made if the number of macules at detection was determined by the age at onset rather than by the delay between onset and detection, younger patients being more prone to develop only one macule. This can be refuted by the fact that the several-macule contacts are younger than the single-macule index patients. The significance of this observation is further enforced by its consistency throughout the study period. The proportion of single-macule among newly detected cases may thus be considered as an indicator of early diagnosis. Even if valid as an indicator, the proportion of single-macule patients is worth reporting only if it is more sensitive than the proportion of disabled patients.

Because disabilities take a long time to develop, the proportion of patients with disabilities can evidence changes in detection activities only after a long delay. Moreover, after many years, the 
group of disabled patients is usually so small that minimal random variations in their number lead to great changes in the proportion.

In Table 1, we observe that the difference of mean age between several- and single-macule patients is much smaller than the difference observed between patients with and without disabilities. In Table 3, the proportion of disabled among contacts is two times less than among index cases, due to contacts being examined more frequently. On the contrary, the proportion of single-macule patients is almost similar for both groups. These two observations are consistent with a shorter delay for the development of several macules than the delay needed to experience disabilities. Thus it seems that the proportion of single-macule patients among newly detected cases is a more sensitive indicator of precocity of detection than the proportion of patients with disabilities. The proportion of single-macule patients would be an especially helpful indicator for programmes running for several years, when it becomes difficult to observe significant variations of the proportion of patients with disabilities.

There are however two difficulties attached to the use of an indicator based on the number of macules. First, a more careful clinical examination, and thus a more experienced staff, is needed to assess the number of macules than to detect disabilities. Second, it is based on paucibacillary patients only. However, if detection occurs progressively earlier for paucibacillary leprosy, it seems only logical to think that it evolves likewise for multibacillary leprosy.

\section{PROGNOSIS}

The second objective of the study was to evaluate the prognosis value of the number of macules at detection on inactivation and relapse probabilities.

Figure 3 shows that single-macule patients inactivate sooner than several-macule patients. The proportion of self-healing cases is probably higher in the first group, but that does not modify the prognosis value of the number of macules. It is however quite satisfactory to observe that regularity to treatment is a better predictor of early inactivation than the number of macules. However, one limitation of the study is the insufficient power of discrimination of the classification used for the macules-several macules just means 'more than one'. It is possible that a more detailed classification would give more gradual and convincing differences in inactivation probabilities. Figures 4 and 5 show that the number of macules is an important predictor of relapse probability, more so than the regularity to treatment. The number of macules at detection depends on, at least, two factors: the delay between onset of the disease and its detection; and the immune def ences of the patients. While several-macule patients all have relatively poor immunity, the single-macule patients include those with poor immunity but early detected, together with patients who have good immunity. That could explain why, their immune defences on average being better, patients with single macule seem relatively protected against relapses. Regarding inactivation, though, the immune status has less influence on the necessary duration of treatment than the compliance to treatment.

The implications of these observations on multidrug therapy (MDT) are certainly worth studying. If the same results were observed after MDT, the selection of the MDT regimen and its duration should then be based not only on bacteriological, but also on clinical criteria.

\section{Acknowledgments}

This study was supported by Amici di Raoul Follereau, Association Française Raoul Follereau, Damien Foundation Belgium, Fondation Luxembourgeoise Raoul Follereau, and Le Secours aux Lépreux, Canada (Member Associations of ILEP). 


\section{References}

${ }^{1}$ Lechat MF, Misson CB, Sansarricq H, Declercq E, Vanderveken M. Omslep recording and reporting system for leprosy patients. Third edition, 117 pp. School of Public Health, Catholic University of Louvain, Brussels, 1987.

2 Lee ET. Statistical methods for survival data analysis. p. 88-95. Lifetime learning publication, Belmont, California, 1980.

3 Rao PSS, Christian M, Jesudasan K, Panniker VK. Time trends in the deformity rate among newly registered patients in Gudiyatham Taluk. In Proceedings of the XIIth International Leprosy Congress, New-Delhi, 20-5 February 1984.

4 Report of activities for 1955-62, Leprosy Centre, Polambakkam.

5 WHO Technical Report Series, No. 716. Epidemiology of le prosy in relation to control. Report of a WHO study group, 1985.

6 WHO Technical Report Series, No.459. Fourth report of the WHO Expert Committee on Leprosy, 1970. 